\title{
Modification of Step in Difficult Laparoscopic Cholecystectomy to Minimize the Conversion Rate
}

\author{
Deepak Verma*, Nemi Chand, Sarthak Sharma, Mahesh Malani, Parul Yadav
}

Department of General Surgery, Dr. Sampurnanand Medical College, Jodhpur, Rajasthan, India

Email address:

drdeepakv3@gmail.com (D. Verma)

${ }^{*}$ Corresponding author

\section{To cite this article:}

Deepak Verma, Nemi Chand, Sarthak Sharma, Mahesh Malani, Parul Yadav. Modification of Step in Difficult Laparoscopic

Cholecystectomy to Minimize the Conversion Rate. Journal of Surgery. Vol. 8, No. 4, 2020, pp. 118-122. doi: 10.11648/j.js.20200804.14

Received: April 26, 2020; Accepted: June 19, 2020; Published: July 4, 2020

\begin{abstract}
Gall stone disease is a common gastrointestinal surgical problem and symptomatic patients needs appropriate and timely treatment in the form of cholecystectomy else the disease process becomes complicated because of recurrent chronic infection, dislodgement of stone to common bile duct, acute infection and more severe complications like perforation of gall bladder. In recent years, Laparoscopic Cholecystectomy is considered as gold standard treatment for symptomatic as well as for few categories of asymptomatic cholelithiasis. Reduced postoperative pain, early return of mobility and to work, cosmetic results and brief hospital stay are distinct advantages of the procedure. Laparoscopic Cholecystectomy becomes difficult in patients with male sex, age above 50 years, obese, history of previous hospitalization due to attacks, previous abdominal surgery scar, palpable gall bladder, impacted stone, overdistended/contracted gall bladder. Various scoring systems have been reported to predict difficult laparoscopic cholecystectomy preoperatively. These difficult cases required longer operating time and hospital stay. The incidence of postoperative complications are also higher as compared to easy Laparoscopic Cholecystectomy Of the total 430 cases included in this study, $200(46.5 \%)$ cases were difficult cases. For performing Laparoscopic Cholecystectomy in these patients successfully i.e. with minimum conversion to open, certain modifications in the classical four-port technique were done. As a result, conversion rate in difficult laparoscopic cholecystectomy could be limited to $7 \%$ only. Postoperative complication were pyrexia (21.5\%), paralytic ileus $(9.5 \%)$, bile stained subhepatic discharge $(6.5 \%)$, port site infection $(8 \%)$ and respiratory complications $(8 \%)$. Late complication was port-site hernia (0.5\%). Operating time 58.7+12.4 minutes in difficult laparoscopic cholecystectomy as compared to $36.5+9.2$ minutes in easy cases and postoperative hospital stay was of $6.9+1.8$ days against $2.3+0.8$ days as compared to easy cases. Unclear anatomy ( $3 \%)$, haemorrhage (4\%), dense adhesions (2\%) and anaesthesia issue (1\%) were the reasons of conversion to open cholecystectomy. It is concluded from this study that preoperative difficult laparoscopic cholecystectomy can be predicted and modifications of the steps of four-port cholecystectomy can be done to minimize the conversion rate.
\end{abstract}

Keywords: Cholelithiasis, Cholecystectomy, Difficult Laparoscopic Cholecystectomy, Conversion to Open Cholecystectomy

\section{Introduction}

Cholelithiasis is a common health problem. All symptomatic and certain categories of asymptomatic gall stones patients need surgical removal of gall bladder i.e. cholecystectomy. The management of cholelithiasis has improved after the introduction of Laparoscopic Cholecystectomy [1]. Laparoscopic Cholecystectomy has many advantages over open approach such as decreased postoperative pain, early return of mobility, better cosmetic result and early return to work. Thus, Laparoscopic Cholecystectomy is now considered the gold standard for treatment of cholelithiasis and has replaced traditional open approach $[2,3]$.

With increasing experience of Laparoscopic Cholecystectomy, selection criteria of patients are widening and what was considered as contraindications of Laparoscopic Cholecystectomy is limited. Presently, bleeding diathesis, carcinoma of gall bladder and patient not fit for general anesthesia are absolute contraindications of Laparoscopic 
Cholecystectomy [4]. The laparoscopic operative procedure may be simple but certain factors like age $>50$ years, male sex, high BMI, history of previous abdominal surgery or common bile duct stenting, local signs of acute cholecystitis, over distended/contracted/ thick walled gall bladder, impacted stone at neck and presence of pericholecystic odema/ fluid makes the procedure difficult [5].

Difficult laparoscopic cholecystectomy not only takes longer operative time but is associated with higher rate of postoperative complication, longer hospital stay and frequent conversion to open cholecystectomy. It is important to identify difficult laparoscopic cholecystectomy preoperatively so that patient and relatives can be counselled and availability of extra resources can be planned and arranged. Conversion rate in Laparoscopic Cholecystectomy varies from 2 to $23 \%$ in previous studies and unclear anatomy and bleeding are two common reasons of converting Laparoscopic Cholecystectomy into open procedure ${ }^{6}$. Preoperative planning according to the factors making procedure difficult might help in reducing these complications and overall conversion rate.

Present study was performed in difficult cholelithiasis cases depending on preoperative factors ${ }^{5}$ and various measures were taken prior to and during surgery to minimize the conversion rate. Factors leading to conversion were also studied.

Aim of the study:

Various modification done in the standard four-port technique of Laparoscopic Cholecystectomy in difficult cases to minimize the complications including conversion to open cholecystectomy.

\section{Material and Methods}

This study was performed in a surgical unit over a period of three years. All patients admitted with diagnosis of cholelithiasis were subjected to preoperative scoring ${ }^{5}$ to predict easy or difficult Laparoscopic Cholecystectomy. Patients with bleeding diathesis, carcinoma of gallbladder, compromised cardiopulmonary status obviating general anesthesia and patients with associated second surgical problem corrected at same time as Laparoscopic Cholecystectomy (like choledocholithiasis, Umbilical or paraumbilical hernia, recurrent appendicitis and incisional hernia) were excluded from the study. Following preoperative scoring, patients were divided in two groups. Group A cases (Score 1 to 5) were predicted as easy and Group B (score $>5$ ) were considered as difficult cases.

Table 1. Criteria used for scoring. ${ }^{5}$.

\begin{tabular}{llll}
\hline S. No. & Parameter & Limit & Score \\
\hline 1 & Age & 50 years & 1 \\
2 & Sex & Male & 1 \\
3 & BMI & $25-27.5$ & 1 \\
& & 27.5 & 2 \\
4 & History of Hospitalization & Yes & 4 \\
5 & Abdominal scar & Infra-umbilical & 1 \\
6 & Palpable GB & Supra-umbilical & 2 \\
7 & Wall Thickness & Yes & 1 \\
8 & Pericholecystic Collection & 4 mm & 2 \\
9 & Impacted Stone & Yes & 1 \\
\hline
\end{tabular}

Total Maximum score -15 .

All cases were operated by single surgeon to eliminate element of expertise. Procedure was started as standard four port technique with two $11 \mathrm{~mm}$ sub umbilical and epigastric ports and two $6 \mathrm{~mm}$ midclavicular and anterior axillary ports. Depending on the factors making Laparoscopic Cholecystectomy difficult, various modification in the standard technique of Laparoscopic Cholecystectomy were done as follow:

Table 2. Modification in operative steps during difficult laparoscopic cholecystectomy.

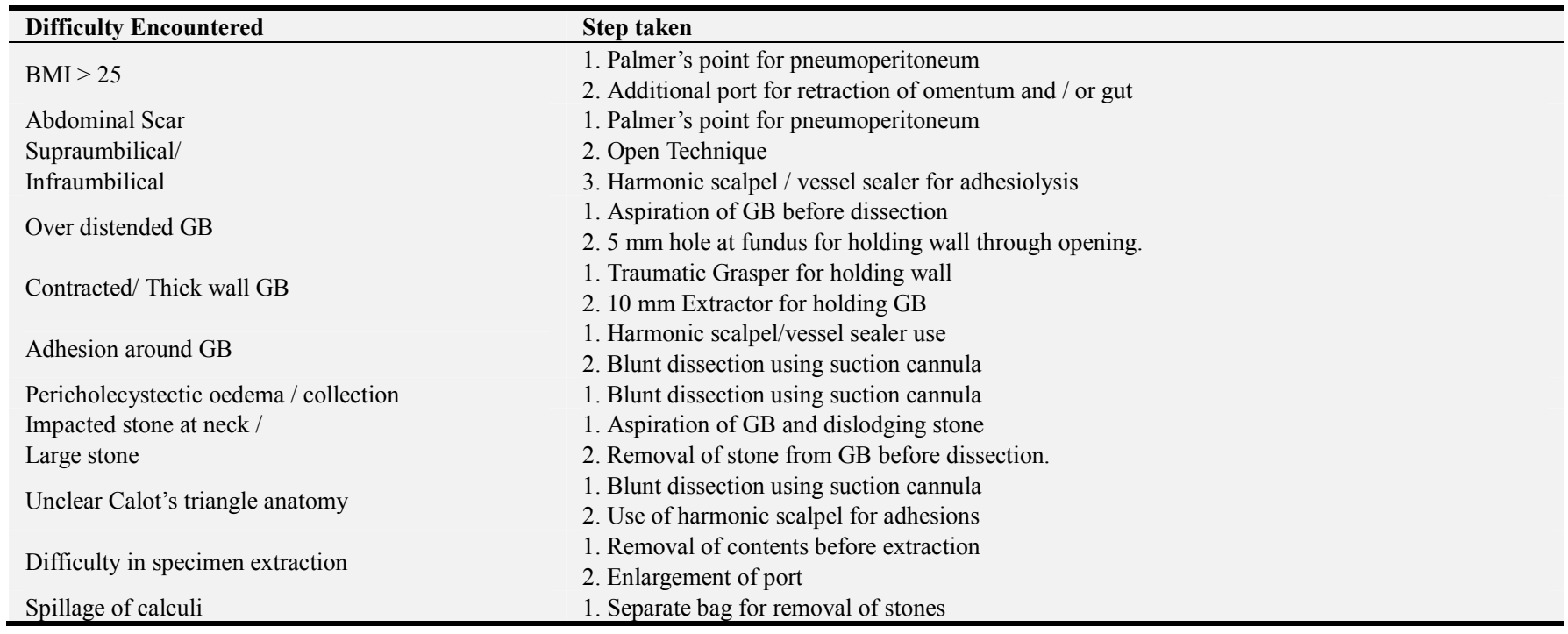

In all difficult cases, sub hepatic drain was inserted after 
with Port closure needle. Nature and amount of drain, postoperative complication, cause of conversion to open were studied. Operating time, postoperative hospital stay and conversion rate was statistically compared with easy group. Modular operating room of the institute has 3-D laparoscopic camera set, ultrasonic harmonic scalpel, vessel sealer available and hence used as and when required during the procedure. Student' $t$ ' test was used for statistical calculation.

\section{Results}

Total of 430 cases of calculus cholecystitis were operated by Laparoscopic Cholecystectomy. On the basis of scoring, $200(46.5 \%)$ were classified as difficult laparoscopic cholecystectomy (score $>5$ ) and remaining $230(53.5 \%)$ were labelled as easy Laparoscopic Cholecystectomy (score $<5)$.

Table 3. Various parameters observed in difficult cases.

\begin{tabular}{llll}
\hline S. No. & Parameter & Limit & Number of patients (\%) \\
\hline 1 & Age & 50 years & $89(44.5 \%)$ \\
2 & Sex & Male & $112(56 \%)$ \\
3 & BMI & 25 & $34(17 \%)$ \\
4 & History of Hospitalization & Yes & $108(54 \%)$ \\
5 & Abdominal scar & Infra-umbilical & $44(22 \%)$ \\
6 & Palpable GB & Supra-umbilical & $26(13 \%)$ \\
7 & Wall Thickness & Yes & $34(17 \%)$ \\
8 & Pericholecystic Collection & 4 mm & $82(41 \%)$ \\
9 & Impacted Stone & Yes & $38(19 \%)$ \\
\hline
\end{tabular}

Table 4. Easy versus Difficult laparoscopic cholecystectomy.

\begin{tabular}{|c|c|c|c|c|}
\hline S. No. & Parameter & Group A & Group B & Significance \\
\hline 1 & Number & $230(53.5 \%)$ & $200(46.5 \%)$ & - \\
\hline 2 & Operating Time & $36.5+9.2 \mathrm{~min}$ & $58.7+12.4 \mathrm{~min}$ & $\mathrm{P}<0.0001$ \\
\hline 3 & Postoperative Stay & $2.3+0.8$ days & $6.9+1.8$ days & $\mathrm{P}<0.0001$ \\
\hline 4 & Conversion to open & Nil & $14(7 \%)$ & Significant \\
\hline
\end{tabular}

Table 5. Post-operative Complications in difficult laparoscopic cholecystectomy.

\begin{tabular}{lll}
\hline S. No. & Complication & Number $(\%)$ \\
\hline 1 & Paralytic ileus for $>24$ hours & $19(9.5 \%)$ \\
2 & Post-operative Pyrexia & $43(21.5 \%)$ \\
3 & Bile stained sub hepatic drain & $13(6.5 \%)$ \\
4 & Port-site infection & $16(8 \%)$ \\
5 & Respiratory complications & $16(8 \%)$ \\
6 & Port-site hernia & $1(0.5 \%)$ \\
\hline
\end{tabular}

No mortality was reported in both group.

Table 6. Reasons for conversion to open $(n=14)$.

\begin{tabular}{lll}
\hline S. No. & Reason of conversion & Number (\%) \\
\hline 1 & Bleeding (From GB Bed) & $4(2 \%)$ \\
2 & Dense Adhesion (Previous peptic & $2(1 \%)$ \\
3 & perforation repair) & $6(3 \%)$ \\
4 & Unclear Calot's Triangle & $2(1 \%)$ \\
\hline
\end{tabular}

\section{Discussion}

Laparoscopic Cholecystectomy has been established as gold standard for treatment of symptomatic as well as for selected categories of asymptomatic gall-stone disease [5, 7]. However, with advances of videoscopy ( high definition, 3-D camera and Infrared with fluorescent ICG cholangiography ), development of different types of hand instruments and availability of newer energy sources like ultrasonic or vessel sealer machines, spectrum of indications for Laparoscopic
Cholecystectomy is widening and contraindication are limited to uncontrolled bleeding diathesis, compromised cardio-pulmonary status contraindicating general anesthesia, uncompensated cirrhosis of liver and preoperative diagnosis of carcinoma of gall bladder. Previously mentioned contraindication like acute cholecystitis etc. are no more considered as contraindication ${ }^{4}$.

Many clinical, radiological and laboratory investigation findings are indicative of difficult laparoscopic cholecystectomy [8, 9]. Even many scoring methods depending on clinical and investigative factors have been devised $^{5,10}$. It had been concluded from all these studies that age more than 50 years, male sex, BMI $>25$, history of previous recurrent attacks, previous abdominal surgery, palpable gall-bladder, increased wall thickness, presence of peri-cholecystic fluid and impacted or large stone are associated with difficult laparoscopic cholecystectomy. Male sex had been independently studied for the same ${ }^{11}$. However, leukocytosis, altered liver function tests, CRP levels, comorbid conditions (diabetes mellitus, COPD, congestive failure), previous ERCP, past history of pancreatitis and jaundice have also been studied in predicting difficult laparoscopic cholecystectomy with variable observations.

Total of 430 cases of calculus cholecystitis were subjected to Laparoscopic Cholecystectomy in this study and preoperative scoring was done as per guideline [5]. Patients with score more than five were labelled as difficult laparoscopic cholecystectomy cases and there were 200 $(46.5 \%)$ such cases with maximum score of 9 in this study.

Of these difficult cases, $44.5 \%$ were above 50 years of age, 
$56 \%$ were male, $17 \%$ had BMI more than $25,17 \%$ had palpable gall bladder. $35 \%$ had history of previous abdominal surgery (22\% infraumbilical and $13 \%$ supraumbilical scar). Sterilization, hysterectomy, umbilical / paraumbilical hernia repair and appendicectomy through midline or right paramedian for infraumbilical scar and epigastric / ventral hernia repair, peptic perforation repair, splenectomy and cystogastrostomy for supraumbilical scar were oberved as prior abdominal surgery.

Although the classical four-port technique had been oldest technique of Laparoscopic Cholecystectomy and over the years many alternative techniques like two-port technique [12], SILS cholecystectomy [13] and NOTES [14] have come in vogue but still the four-port technique is most commonly used technique the world over because it is easy to learn, perform and teach. Overall it can be used for almost all kind of cholelithiasis patients whereas the other techniques cannot be used for all cases and when these newer technique fails, ultimately, they are converted to classical technique. It is for this reason that classical four-port technique was used in the present study and the modification were done to minimize the conversion rate in difficult laparoscopic cholecystectomy.

In patients with high BMI and previous abdominal scar, Palmer's point was used for creating pneumoperitoneum and sub umbilical optical trocar was introduced. In patients with scar in this region, open method for trocar insertion was used. All other trocars were placed under vision. Additional $5 \mathrm{~mm}$ trocar was inserted in left mid-clavicular line for retracting omentum and / or other visceras. Ultrasonic machine and vessel sealer were both available in the operating theatre. They were used for adhesiolysis (previous abdominal scar adhesions), to divide vascular adhesions of omentum with gall bladder and dissecting Calot's triangle as and when necessary.

Over distended gall bladder was aspirated. In very thick wall gall bladder, $5 \mathrm{~mm}$ opening was made at fundus and contents were sucked using suction, A $5 \mathrm{~mm}$ traumatic grasper was introduced with one prong inside and other outside $5 \mathrm{~mm}$ opening at fundus of gall bladder for retracting fundus. In contracted gall bladder, anterior axillary port was converted to $10 \mathrm{~mm}$ and $10 \mathrm{~mm}$ extractor was used for holding and retracting fundus.

In difficult Calot's with dense adhesions, suction cannula was used as blunt dissector and posterior dissection was preferred till critical view of safety is seen. In no case, cystic duct or artery was clipped without delineation. In impacted stone at neck, attempt was made to manipulate stone in the body of gall bladder and if failed, incision over neck was given to take out stone and then to dissect the Calot's triangle. In 3\% cases subtotal cholecystectomy was done and neck was closed with polyglactin suture.

Distended gall bladder with multiple stone was subjected to evacuation of stones using stone holding forceps before extraction through epigastric port. In large stone or thickwalled gall bladder, epigastric port was enlarged. All specimens were removed using specimen extraction bags.
With above mentioned planning, the procedure could be completed in $93 \%$ of the difficult laparoscopic cholecystectomy with conversion rate of $7 \%$. Overall conversion rate was $3.2 \%$. Conversion rate varies from 1 to $13 \%$ [8] and higher conversion rate is linked to patients with age above 50 years, male sex [9], obesity [15], Acute cholecystitis [16]. In study of difficult laparoscopic cholecystectomy cases, conversion rate as high as $23.3 \%$ have been reported [17]. In present study, dealing with these factors by modification in four-port technique had been useful in reducing the conversion to open.

Reasons for conversion (Table 6) were unclear anatomy despite all efforts particularly Calot's triangle anatomy in (3\%), bleeding from gall bladder bed during / after dissection of gall bladder $(2 \%)$, dense adhesion hiding gall bladder $(1 \%)$ [having history of previous peptic perforation repair) anesthesia problem in $1 \%$. Although not observed in the present study, CBD injury? Choledochoduodenal fistula, GB mass, failed insertion of trocar and contracted GB had been other reasons for conversion of Laparoscopic Cholecystectomy to open cholecystectomy [18].

Operating time of $58.7+12.4$ minutes in difficult laparoscopic cholecystectomy as compared to $36.5+9.2$ minutes in easy cases and post-operative hospital stay of $6.9+1.8$ days against $2.3+0.8$ days were observed in this study. Both values were statistically significant $(p<0.0001)$.

Post-operative pyrexia $(21.5 \%)$, paralytic ileus for more than 24 hours $(9.5 \%)$, port site (specimen extraction port) $8 \%$ were various complication observed. $6.5 \%$ patients had bile stained drain output from sub hepatic space which gradually subsided except in $2(1 \%)$ patients. Possibly the gall bladder bed was the source of bile stained drain in majority of cases. Postoperative ERCP (performed for continuing bile stained drain) revealed a small calculus at lower end of CBD which remained undetected preoperatively. Stone extraction and stent placement was done to resolve the complication. Portsite infection also responded to conservative treatment but 1 $(0.5 \%)$ patient had port-site hernia. Respiratory complications $(8 \%)$ also responded to conservative therapy. However, no mortality was reported in this study. Similar complications have been reported in literature [18].

However, open cholecystectomy still has a place in the era of laparoscopy and is indicated in selected cases like patients with liver cirrhosis, Mirizzi gall bladder mass and anesthetic problem without laparoscopic trial [18]. Hence all general surgeons should have adequate exposure to open cholecystectomy technique not only for conversion during laparoscopic cholecystectomy but for management of such cases. Conversion to open or planning case as open cholecystectomy without laparoscopic attempt is not a failure but a correct decision taken in view of patients' safety. However, conversion to open is associated with postoperative pain, prolonged paralytic ileus and hospital stay, increased chances of respiratory and wound complication and hence sincere effort by all measures to complete cholecystectomy laparoscopically as far as possible must be made with safety of patient in mind. 


\section{Conclusion}

It can be concluded from the present study on 200 cases of difficult laparoscopic cholecystectomy that if preoperative difficulties are defined using scoring method, we can modify certain steps in classical four-port laparoscopic cholecystectomy and prepare OR beforehand. The suggested modifications can minimize the conversion rate in these cases to as low as $7 \%$ without increasing the complications.

\section{Conflict of Interest}

The authors declares that they have no competing interest.

\section{References}

[1] Polychronidis A, Laftsidis P, Bounovas A et al (2008) Twenty years of Laparoscopic Cholecystectomy: Philippe Mouret, March 17, 1987, JSLS 12: 109-111.

[2] McMahon AJ, Fischbacher CM, Frama SH et al (2008) Impact of Laparoscopic Cholecystectomy: a population based study. Lancet 356: 1632-1637.

[3] Ercan M, Bostanci EB, Teke Z et al (2010) Predictive factors for conversion to open surgery in patients undergoing elective Laparoscopic Cholecystectomy. J Laparoendosc Adv Sur Tech 20, 427-434.

[4] Lajan J, Parilla P, Rables R et al (1998) Laparoscopic Cholecystectomy v/s open cholecystectomyin the Treatment of Acute Cholecystitis. Arch Surg 133 (2), 173-5.

[5] Randhawa JS, Pujahari AK (2009) Preoperative prediction of difficult laparoscopic cholecystectomy: a scoring method. Indian J Surg (July-August) 71: 198-201.

[6] Tong B, Cuschieri A (2006) Conversion during Laparoscopic Cholecystectomy: rosk factors and effects on patient outcome. J Gastrointest Surg 10 (7): 1081-1091.

[7] Lam CM, Murray PE, Cuscheri A (1996) Increased cholecystectomy rate after the introduction of Laparoscopic Cholecystectomy in Scotland Gut 38: 282-284.
[8] Nidoni R, Udachan TV, Sasnur P et al (2015) Predicting difficult laparoscopic cholecystectomy based on clinicoradiological assessment J Clin Diagn Res 9 (12): PC09PC12.

[9] Kanaan SA, Murayama KM, Merriam LT et al (2002) Risk factors for conversion to laparoscopic to open cholecystectomy J Surg Res Jul; 106: 20-24.

[10] Joshi MR, Bohara TO, Rupakheti S et al (2015) Preoperative prediction of difficult laparoscopic cholecystectomy J Nepal Med Assoc 53 (200): 221-226.

[11] Hong-Hui Lein MD, Ching-Shui Huong (2002) Male gender. Risk factor for severe symptomatic cholelithiasis. World $\mathrm{j}$ Surg 26: 598-601.

[12] Lee KW, Poon CM, Leung KF et al (2005) Two-port needlescopic cholecystectomy: prospective study of 100 cases. Hong Kong Med J. Feb; 11 (1): 30-35.

[13] Borutan AG, Klein D, Kilian M et al (2020) Long-term follow-up after single incision laparoscopic surgery. Surg Endos, Jan; 34 (1): 126-132.

[14] Bulian DR, Knuth J, Lehmamm KS et al (2015). Systematic analysis of the safety and benefits of transvaginal hybrid NOTES cholecystectomy. World J Gastroenterol, Oct 14; 21 (38): 10915-23.

[15] Fried GM, Borkun JS, Sigman HH et al (1994) Factors determining conversion to laparotomy in patients undergoing Laparoscopic Cholecystectomy. Am J Surg 167: 35-41.

[16] Rosen M, Brody F, Ponsky J (2002) Predictive factors for conversion of Laparoscopic Cholecystectomy. Am J Surg 184: 254-258.

[17] Lal P, Agarwal NP, Malik VK, Chakarvati AL (2002) A difficult laparoscopic cholecystectomy that requires conversion to open procedure can be predicted by preoperative ultrasonography. JSLS Jan-Mar 6 (1): 59-63.

[18] Nakeeb A EI, Mahdy Y, Salem A et al (2017) Open Cholecystectomy has a place in the Laparoscopic Era: a retrospective cohort study. Inj J Surg 79 (5): 437-443. 